\title{
LOCALIZATION OF SEIZURE SOURCES USING BLIND IDENTIFICATION AND A NEW CLUSTERING ALGORITHM
}

\author{
Delaram Jarchi ${ }^{1}$, Mohammad Taheri ${ }^{1}$, Reza Boostani ${ }^{1}$, Saeid Sanei ${ }^{2}$ \\ ${ }^{1}$ Department of Computer Science and Engineering, Shiraz University, Shiraz, Iran \\ ${ }^{2}$ Centre of Digital Signal Processing, Cardiff University, Cardiff, UK
}

\begin{abstract}
The In this paper, a new method is proposed to localize the seizure sources from multi-channel electroencephalogram (EEG) signals. The second order blind identification (SOBI) is applied to estimate the brain source signals in a number of signal segments. After calculating the unmixing matrices in several time frames, the rows of these matrices are clustered by a new proposed clustering method. By multiplying each cluster center to the electrode signals (EEGs), the brain signal sources are approximated. According to short term largest lyapunov exponent values, the main seizure source signal is separated from the others. This source signal is projected back to the electrodes' space in order to localize the seizure source in the brain. The simulation results and also the clinical tests derived using the simultaneous intracranial recordings verify the accuracy of the system.
\end{abstract}

Keywords - Seizure, SOBI, Clustering, localization, BSS

\section{INTRODUCTION}

One of the most common neurological disorders is seizure which affects more than sixty million people in the world. EEG signals, PET and fMRI images are the most commonly used data for the evaluation of brain activity and neurological abnormalities [6] but most analysis of epilepsy is based on the EEG because the recording procedure is much cheaper than those of the fMRI and PET. Several blind source separation (BSS) have been applied to the raw EEG signals for extracting the main brain sources. In this study, the wellknown SOBI algorithm is used because it makes fewer assumptions on the statistical properties of the data than other BSS algorithms. Before applying SOBI, the number of sources is estimated in each time frame [7].

In this research, EEG signals from 3 subjects with focal seizure are recorded. The algorithm for extracting the brain sources includes two stages [11]; First, SOBI algorithm is applied to different time frames of each channel to extract a number of sources in each segment. Then, our novel clustering method is used to cluster the rows of the unmixing matrices calculated in different frames. After separating the brain sources, it is possible to detect the seizure source by calculat- ing short term largest lyapunov exponent $\left(S T L_{\max }\right)$ [9] values for all the sources. The selected seizure source is projected back to the electrodes. The projected source is used to locate the seizure foci. To verify our method, the corresponding intracranial signals are recorded invasively which show the exact location of foci. The accuracy of the method has also been tested by simulation.

The remainder of this paper is structured as follows. In section 2, the SOBI algorithm is briefly described and in section 3 , a new clustering method is proposed. Then in section 4, the seizure source localization procedure is described. In section 5 the results for both real and synthetic data are shown. Finally, section 6 concludes the paper.

\section{SECOND ORDER BLIND IDENTIFICATION}

Second order blind identification (SOBI) is able to estimate the mixing matrix as long as the unknown source signals are assumed temporally uncorrelated to each other. But, they have non-zero time-delayed autocorrelations. This is a plausible assumption for the case of EEG and electroculogram (EOG) sources [8].

$\mathbf{x}(t)=\left[x_{1}(t), \cdots, x_{m}(t)\right]$, where $m$ denotes the number of electrodes. Each $x_{i}(t)$ is assumed to be a linear mixture of some unknown sources $s_{i}(t)$ via an unknown mixing matrix A.

$$
\mathbf{x}(t)=\mathrm{A} \mathbf{s}(t)
$$

Where $\mathbf{s}(t)$ contains the source signals and $\mathrm{A}$ is the mixing matrix. The SOBI uses only $\mathbf{x}(t)$ to produce an unmixing matrix $\mathbf{W}$ which approximates $\mathrm{A}^{-1}$, subject to scaling and permutation of the separated sources. Therefore, the estimated sources can be obtained by the following formula:

$$
\hat{\mathrm{s}}(\mathrm{t})=\mathbf{W} \mathbf{x}(\mathrm{t}), \quad \mathbf{W}^{-1}=\mathbf{P D} \hat{\mathbf{A}}
$$

Where $\mathbf{P}$ and $\mathbf{D}$ are respectively permutation and scaling matrices. The SOBI computes the mixing matrix as the matrix that jointly diagonalizes [10] a set of $p$ cross correlation ma$\operatorname{trices} R\left(\tau_{i}\right)=E\left[x(t) x\left(t-\tau_{i}\right)^{T}\right]$, where $i=1, \ldots, p$, and $E[\cdot]$ is the expectation operator. In this paper we use $p=$ 
$\left\lfloor L_{a} / 3\right\rfloor$ where $L_{a}$ is the number of EEG data samples in the analyzed data frame and $L$.$\rfloor denotes the floor operator.$

\section{CLUSTERING THE ROWS OF UNMIXING MATRICES}

In this section a novel technique is introduced to initialize the centers of clusters. After initializing the cluster centers, two new criteria are proposed for splitting and merging. Then a restorative technique based on neighborhood information is proposed. Finally, the flow chart of the clustering system that uses techniques described in this paper is depicted in Figure 2. This system uses DSRPCL2 [1] as the main method.

\subsection{INITIALIZATION OF CLUSTER CENTERS}

In this paper the clustering method is used as a core of the system. The performance of the system however, is considerably improved by introducing some extensions to the main process as follows. Up to now, some methods have been proposed to initialize the cluster centers such as variable division and leader algorithm [2]. Our experiments show that the more distance between initial centers, the more chance to find the best final clusters. To comply with this requirement, the method proposed by Mico [3] is implemented.

From now on, $W^{\rho}$ represents the $\rho^{\text {th }}$ initial point as the cluster center. This method chooses these points from instances of sample data. At the first, $W^{l}$ is chosen randomly from instances and then other points are selected by :

$$
W^{\rho}=\underset{x \in\left(\mathrm{M}-B_{\rho}\right)}{\arg \max }\left(\sum_{j=1}^{\rho-1} f\left(x, W^{j}\right)\right) \rho=2,3, \ldots, K
$$

where, $M$ is the set of instances of sample data, $B_{\rho}=\left\{W^{1}, W^{2}, \ldots, W^{\rho-1}\right\}$ is the set of chosen instances and $K$ is the number of initial clusters, $f(a, b)$ is a distance function between $a$ and $b$. Here, Euclidean distance function is used as $f$. Moreover, in each step, one unselected instance with the largest summation of distances from selected ones is chosen as the next point. But this method seems to be inefficient if $K>2 d$ for a $d$-dimensional feature space. As a simple example, in a one dimensional space depicted in Figure 1, after selecting A and B as two instances with the largest distance between them, these two points have a same distance summation. Then, the question is what point is the best.

Therefore, equation (3) is changed to:

$$
W^{\rho}=\underset{x \in(\mathrm{M}-B}{\arg \max }\left(\sum_{j=1}^{\rho-1} \sqrt{f\left(x, W^{j}\right)}\right) \quad \rho=2,3, \ldots, K
$$

With this approach, in Figure 1, the point with the most similar distance from $A$ and $B$ is chosen as the next point.

\subsection{NEW SPLITTING AND MERGING- BASED APPROACH}

The main motivation of ISODATA [4] is to improve the KMeans method by using auxiliary techniques such as merging or splitting. ISODATA clustering method uses special criteria and suffers some lacks to detect correct clusters. Here, a novel method based on merging and splitting is proposed to overcome this problem.

\subsubsection{MERGING CONDITION}

In ISODATA, if the centers of two clusters are nearer than a specified threshold, the main condition for merging is satisfied. This criterion doesn't consider other properties of the clusters such as their size and distributions. A novel criterion is introduced here which is based on the expected internal distances of a cluster.

$$
D_{M S R}^{A}=\underset{X^{n} \in A}{\operatorname{Mean}}\left(f\left(X^{n}, S N^{n}\right)\right)
$$

where, $D_{M S R}^{A}$ is the $D_{M S R}\left(D_{M S R}\right.$ that is the Mean of Solitude Radiuses of instances belonged to the specified cluster) of cluster A and $S N^{n}$ is the nearest instance to $X^{n}$ such that both of them belong to the same cluster. Hence, each instance in a cluster is expected to be at $D_{M S R}$ from its nearest cluster-mate. Therefore, a criterion is proposed based on the external distances of two clusters:

$$
\begin{gathered}
\qquad D_{\text {min }}(A, B)=\min _{X^{a} \in A, X} b_{\in B} f\left(X^{a}, X^{b}\right) \\
\text { Where } D_{\text {min }}(A, B) \leq \beta \times \max \left(D_{M S R}^{A}, D_{M S R}^{B}\right)
\end{gathered}
$$

With these definitions, the merging condition is proposed by (7). $\beta$ is a constant and considered 1.5 in this paper.

\subsubsection{SPLITTING CONDITION}

Suppose $V=\left\{V^{l}, V^{2}, \ldots, V^{d}\right\}$ is a set of vectors in $d$ dimensional feature space such that each $V^{i}$ and $V^{j}$ are perpendicular. Principle component analysis (PCA) [5] method produces $V$ by computing the eigenvectors of covariance matrix of instances. To check the splitting condition of a cluster, $V$ is computed for its corresponding instances. Then, the instances are projected onto each vector separately. After projection, the cluster is considered in $d$ independent directions. Splitting condition is satisfied for a specified cluster if one of its projections is qualified to be split. In this case, each instance has only one value associated to its unique feature. Then the instances are sort based on this feature value. Each one dimensional cluster is qualified to be split if the distance between the two consequent instances is more than a threshold which is $\beta$ times greater than $D_{M S R}$ of the original cluster. $\beta$ is the constant used in (7).

\subsection{K-NEAREST NEIGHBOR RESTORATION}

Regardless of which clustering method has been used as the core, according to the complexity of dataset, some methods 
may make some mistakes about a few samples of data located at the boundary of clusters. In this paper, a new technique is used to overcome this problem.

After reaching the stop condition of the main method, all the instances belonged to a cluster are labeled uniquely. Finally a post-processing technique based on KNN [2] method is suggested to overcome the lack of producing small extra clusters. In each step, one instance of sample data is chosen as a new sample in KNN method and other ones assumed to be the training data with their associated labels. Hence, KNN overwrites the label of the selected instance and this process goes on for all the sample data. This post-processing is performed on the instances iteratively until no change in labels is occurred.

\section{SEIZURE LOCALIZATION}

In this stage after clustering the rows of the unmixing matrices deriven by the SOBI algorithm in several time frames, we can form a new unmixing matrix named $U$ which each row is the resulted cluster centers. Obviously, $U$ is not square (as far as the number of EEG recording electrodes is reasonably high it is expected to have a fewer number of sources than sensors), hence, we can not use its inverse to do source projection. To solve this problem the following stages are performed in order to project back the seizure source signal to the electrodes:

Stage 1) Compute the unmixing matrix called $Q$ over the whole specified number of time frames where the number of sources is set equal to the number of electrodes.

Stage 2) For the seizure source, we compare its corresponding row in matrix $U$ to the rows of the estimated unmixing matrix from stage 1 . This can be carried out by computing the correlation coefficient between the equivalent row of the seizure source in matrix $U$ and each rows of $Q$ according to the following equation:

$$
r=\frac{\sum_{m}\left(A_{m}-\bar{A}\right)\left(B_{m}-\bar{B}\right)}{\sqrt{\left(\sum_{m}\left(A_{m}-\bar{A}\right)^{2}\right)\left(\sum_{m}\left(B_{m}-\bar{B}\right)^{2}\right)}}
$$

where $A$ is a row vector of $\mathrm{U}$ which after multiplying by electrode signals leads to generation of the seizure source and $\bar{A}$ is the mean of $A$ entries. $B$ is a row vector of $\mathrm{Q}$ and $\bar{B}$ is the mean of $B$ entries.

Stage 3) Find the row index of $\mathrm{Q}$ which generates the maximum $r$. Keep the seizure source and set the rest of the outputs to zero.

Stage 4) Compute $\mathrm{Z}=\mathrm{Q}^{-1} \mathrm{Y}$, where $\mathrm{Z}$ is the projection of seizure source onto the electrodes' space. The method of dipole localization [12] is then applied by exploiting this projection in order to localize the source in the $3 \mathrm{D}$ head space.

\section{SIMULATION AND EXPERIMENTAL RESULTS}

To show the effectiveness of our proposed method, this approach is applied to the real and simulated (synthetic) data.

\subsection{SYNTHETIC DATA}

For the synthetic data, four electrodes (Fp1,Fp2,F7,F8) are selected. These electrodes record mixture of the sources inside the head. To synthesis the EEG signals for different electrodes for a patient with focal seizure, the main assumption is that the electrode signals are generated from two main sources. One source is the noise while the other source is a sinusoidal that is generated via the following equation:

$$
\operatorname{Sin}(x)=\operatorname{Sin}(\pi / 2 x)+\operatorname{Sin}(\pi / 4 x)+\operatorname{Sin}(3 \pi / 2 x)
$$

The mixing matrix is inversely proportional to (square of) the distances from each source to all the electrodes. After constructing the mixing matrix, electrode signals can be generated. The results of localization using two hybrid systems are illustrated in Table 1.

Table 1- The Localization error of two hybrid methods.

\begin{tabular}{|c|c|c|}
\hline \multirow{2}{*}{$\begin{array}{c}\text { SOBI + } \\
\text { K-Mean }\end{array}$} & $\begin{array}{l}\text { Localized } \\
\text { source's axe }\end{array}$ & {$\left[\begin{array}{lll}48.84 & 22.01 & 1.788\end{array}\right]$} \\
\cline { 2 - 4 } & MSE & 0.1245 \\
\hline $\begin{array}{c}\text { SOBI + The proposed } \\
\text { clustering algorithm }\end{array}$ & $\begin{array}{l}\text { Localized } \\
\text { source's axe }\end{array}$ & {$\left[\begin{array}{lll}62.48 & 43.23 & -0.2723\end{array}\right]$} \\
\cline { 2 - 4 } & MSE & 0.0797 \\
\hline
\end{tabular}

\subsection{REAL DATA}

In this part scalp EEG signals of 3 patients with focal seizure are examined. Each EEG signal recordings takes 2 minutes long with sample frequency of $200 \mathrm{~Hz}$ from 16 channels:

FP1, FP2, F3, F4, C3, C4, P3, P4, O1, O2, F7, F8, T3, T4, T5, T6.

The SOBI with the given number of sources in each time frame is applied to the eight time frames from 40 seconds to 80 seconds [11]. Then, the new auxiliary clustering method is applied to the rows of the unmixing matrices of all the time frames. After the clustering process, each cluster center is multiplied by the EEG signals to find the estimated sources. Our main interest is to find the location of the seizure source, and it is clear that for a focal seizure, the projection of seizure position on the scalp remains in the same place and cluster center of seizure source can give us the main seizure source signal. The resulted sources are shown in Figure 3. $S T L_{\max }$ values have been measured from the second signal of Figure 3 (from the top). The $S T L_{\max }$ values show a drop around $14^{\text {th }}$ second. Therefore, we can consider this signal as the main seizure source. The results of applying the four stages above in order to localize the seizure source is shown in Figure 4. Dipole plot of the seizure source is shown in Figure 5.

\section{DISCUSSION AND CONCLUSION}

A new hybrid method for clustering the row vectors of the SOBI unmixing matrix has been developed in this paper. The outcome of this mathematical derivation has been effectively used in identification of seizure source signals from the EEGs. A preprocessing stage is required to estimate the number of sources whereby the number of clusters is identified. 
In a post-processing stage the estimated seizure source is backprojected to the electrodes' space and used for localization of that source. The result of using the new clustering technique has also been compared to those of the conventional k-mean algorithm. The carried out experiments together with clinical inspections verify the accuracy of the system.

\section{REFERENCES}

[1] Rasmussen, E., Clustering Algorithms., editors, Frakes, W.B., Yates, R. Baeza., "Information Retrieval Data Structures and Algorithms", Englewood Cliffs, Prentice Hall, 1992, pp. 419-42.

[2] Wiley, John, Ltd., Sons, Statistical Pattern Recognition, second edition, Webb, Andrew R., Ltd, QuinetiQ, Malvern, UK, Copyright 2002.

[3] Mic'o, M.L., Oncina, J., Vidal, E., "A new version of the nearestneighbour approximating and eliminating search algorithm (AESA) with linear preprocessing time and memory requirements", Journal of Pattern Recognition, Vol. 15, No. 1, pp. 9-17, 1994.

[4] Ball, G.H., Hall, D.J., ISODATA, "A Novel Method of Data Analysis and Classification", Technical report, Stanford University, USA, 1965.

[5] Lindsay, I. Smith, A Tutorial on Principal Components Analysis, 2002, it is available at http://csnet.otago.ac.nz .

[6] J. Corsini, L. Shoker, S. Sanei, and G. Alarcón, "Epileptic Seizure Predictability From Scalp EEG Incorporating Constrained Blind Source Separation", IEEE Transactions on biomedical Engineering, Vol. 53, No. 5, May 2006.

[7] X. Bai and B. He, "Estimation of Number of Independent Brain Electric Sources From the Scalp EEGs", IEEE Transactions on biomedical Engineering, vol. 53, No. 10, October 2006.

[8] A. Belouchrani, K. Abed-Meraim, J.-F. Cardoso, and E.Moulines "A blind source separation technique using second-order statistics", IEEE Transactions on Signal Processing., Vol. 45, No. 2, pp. 434-444, 1997.

[9] L. D. Iasemidis, J. C. Principe, and J. C. Sackellares, "Measurement and quantification of spatio-temporal dynamics of human epileptic seizures", in Nonlinear Biomedical Signal Processing, M. Akay, Ed. Piscataway, NJ: IEEE Press, 2000.

[10] J.-F. Cardoso and A. .Souloumiac, "Jacobi angles for simultaneous diagonalization," SIAM J. Matrix Anal. Appl., Vol. 17, No. 1, pp. 161-164, 1996.

[11] D. Jarchi, R. Boostani, S. Sanei, "Seizure Source Selection via a Hybrid Second Order Blind Identification and Gap Statistic Algorithm", proceeding of IEEE conference on Digital Signal Processing, page(s): 183-186, Cardiff, UK, July 2007.

[12] R. Kavanagh, T. M. Darccey, D. Lehmann, and D. H. Fender, "Evaluation of methods for three-dimensional localization of electric sources in the human brain." IEEE Transactions on Biomedical Engineering, Vol. BME-25, No. 5, pp. 421-429, 1978.

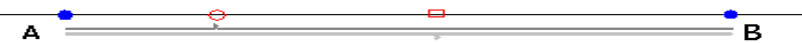

Figure 1 - Selecting initial points in one dimensional space.

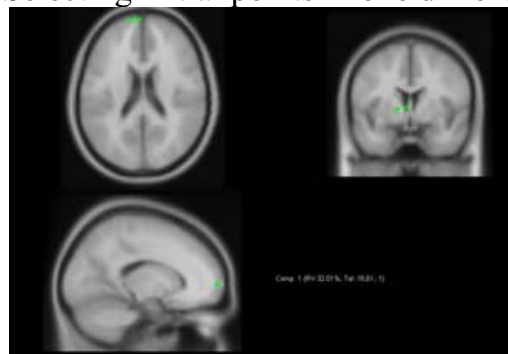

Figure 5 - Dipole plot of seizure component. It can be seen that, as expected, seizure source dipole is localized accurately in the fontal lobe.

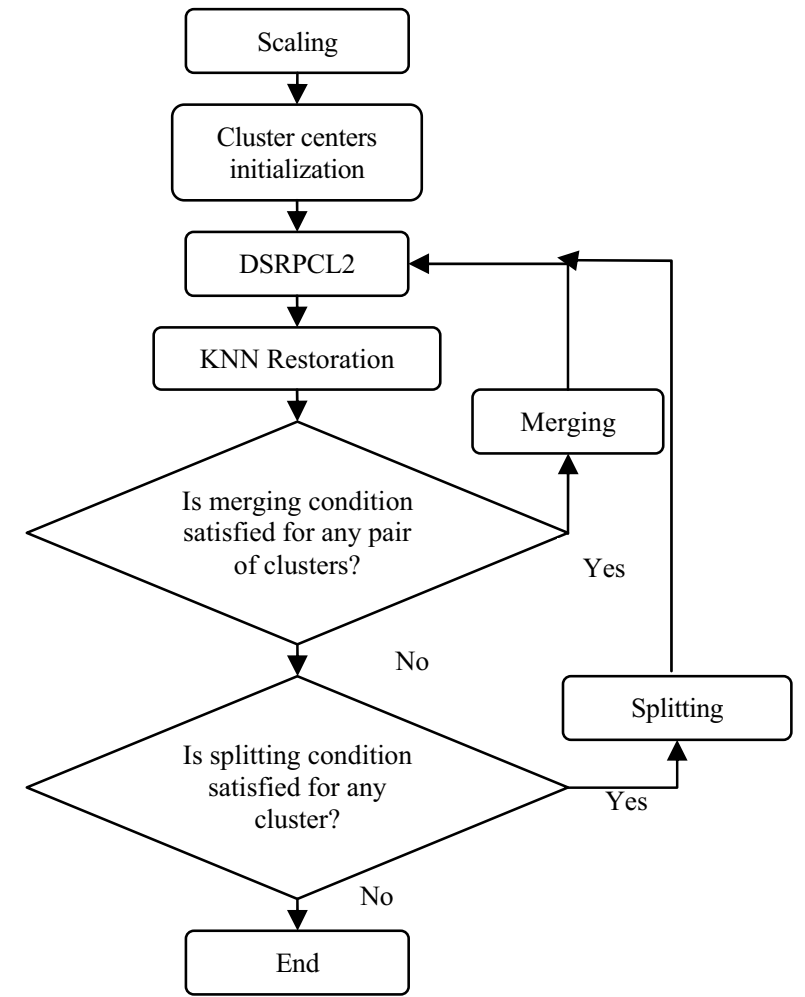

Figure 2 - Block diagram of the combinatorial clustering system

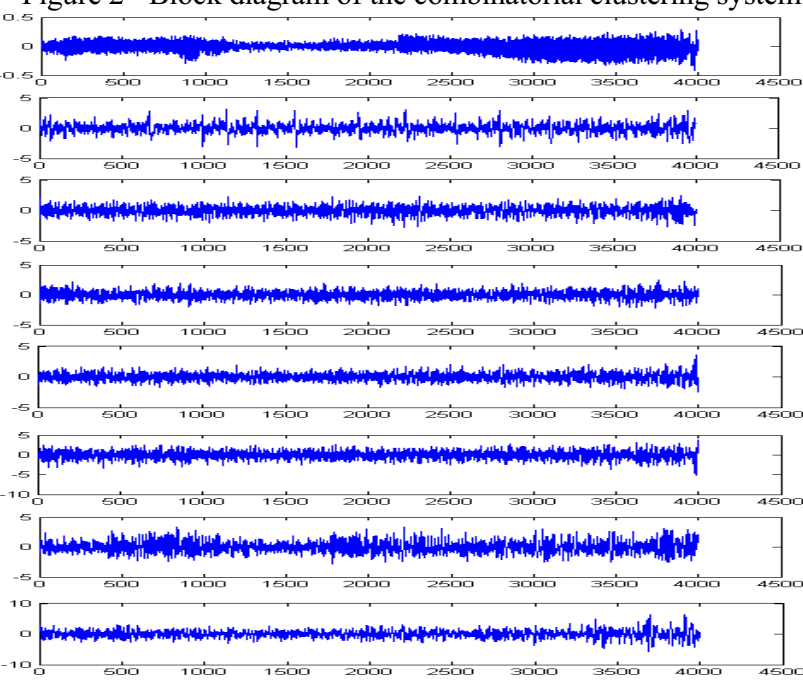

Sample number

Figure 3 - Estimated EEG sources of a patient with focal seizure.

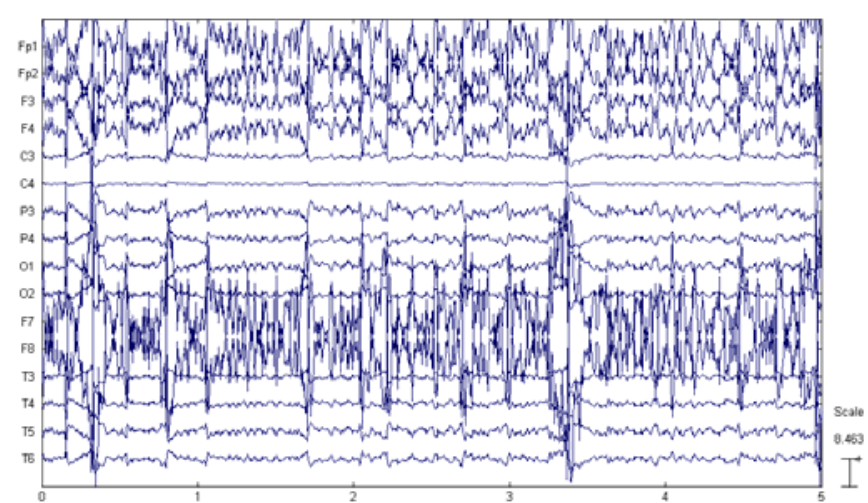

Figure 4 - Projected seizure source to the electrodes. 